\title{
长江口水域鱼卵、仔稚鱼种类组成和数量的分布与变化
}

\author{
张 衡 ${ }^{1,2, *}$, 杨胜龙 ${ }^{1}$, 孟海星 ${ }^{2}$ \\ (1. 中国水产科学研究院东海水产研究所 渔业资源遥感信息技术重点开放实验室，上海２00090; \\ 2. 华东师范大学 河口海岸学国家重点实验室, 上海 200062)
}

\begin{abstract}
摘要：根据 2005 年 4 和 11 月、 2006 年 4 和 9 月在长江河口水域进行的鱼卵、仔稚鱼种类和数量调查, 并结 合 1990 年 9 月和 1991 年 3 月的历史数据, 对该水域的鱼卵、仔稚鱼群落结构的种类组成和数量的季节变化作了 研究, 其结果共发现鱼卵、仔稚鱼 36 种(类), 以海洋鱼类种类最多。秋季航次鱼卵、仔稚鱼的平均种类数和丰度 均高于春季。河口咸淡水区鱼卵总数显著高于淡水区，而仔稚鱼总数略低于淡水区。与 1990-1991 年数据相比, 2005-2006 年春、秋季的鱼卵和仔稚鱼丰度均有所增加。1990、2006 年秋季(9 月)鱼卵、仔稚鱼种类组成差异较 大, 特别是尧嘴红鲌和太湖新银鱼丰度差异较大。这些都说明近 20 年来, 长江河口水域鱼卵、仔稚鱼种类组成和 数量均发生了明显的改变。
\end{abstract}

关键词: 长江河口; 鱼卵; 仔稚鱼; 种类; 年际变化

中图分类号: Q178; S931.5 文献标志码: A 文章编号：0254-5853-(2012)03-0255-06

\section{Distribution and changes in species composition and abundance of ichthyoplankton in the Yangtze estuary}

\author{
ZHANG Heng $^{1,2, *}$, YANG Sheng-Long ${ }^{1}$, MENG Hai-Xing ${ }^{2}$ \\ (1. Key Laboratory of Fisheries Resources Remote Sensing and Information Technology Resources, East China Sea Fisheries Research Institute, \\ Chinese Academy of Fishery Sciences, Shanghai 200090, China; 2. State Key Laboratory of Estuarine and Coastal \\ Research, East China Normal University, Shanghai 200062)
}

\begin{abstract}
Based on four surveys of eggs and larvae in the Yangtze estuary in 2005 (April and November) and 2006 (April and September), combined with the historical data of the wetland in 1990 (September) and 1991 (March), we analyzed seasonal changes in fish species composition and quantity of ichthyoplankton. Thirty-six species of egg and larvae were collected and marine fish species were the highest represented ecological guild. Average fish species and average abundance in spring were lower than in autumn for every survey. The total number of eggs in brackish water was higher than in fresh water, but the total number of larvae and juveniles in brackish water was lower. The abundance of eggs and larvae during from 2005 to 2006 in both spring and autumn was higher compared to those from 1990 to 1991. Obvious differences in species composition in September between 1990 and 2006 were found, especially for Erythroculter ilishaeformis and Neosalanx taihuensis. Fish species composition and quantity within the ichthyoplankton community has obviously changed in the Yangtze estuary over the last 20 years.
\end{abstract}

Key words: Yangtze estuary; Egg ; Larvae; Species composition; Annual change

河口区域由于上游带来的大量营养盐, 在此沉 积或悬浮于水体之中，进而乃育了大量浮游生物， 以致初、次级生产力很高, 成为海洋中的高生产区 (Barnes, 1974; Berasategui et al, 2004)。由于适合仔 稚鱼摄食的饵料生物非常丰富, 加上水浅及浊度高
所造成的良好隐蔽性，使得大部分的捕食者不敢靠 近或难以进行捕食, 所以仔稚鱼在河口水域得以快 速成长且死亡率较低(Deegan, 1990), 因此, 一些海 洋性鱼类、河口定居种和淡水鱼类等大都以河口水 域作为主要的产卵场和育幼场, 早期生活史主要在

收稿日期: 2011-10-26; 接受日期：2012-01-12

基金项目：国家 “863” 计划项目(2007AA092202); 国家自然科学基金项目(31170508); 大洋生物资源开发和利用上海市高校重点实验室开放基 金(KF200908)

*通信作者(Corresponding author), E-mail: zheng615@sina.com.cn 
河口水域度过(Bell et al, 1984; Haedrich \& Haedrich 1974; McErlean et al, 1973), 鱼卵、仔稚鱼的种类和 数量都非常的丰富(Loneragan et al, 1989; McErlean et al, 1973)。

长江河口水域丰富的营养物质为多种经济鱼 类的产卵提供条件, 成为多种仔、稚、幼鱼索饵育 肥的场所(Chen \& Chen, 1999; Huang, 1988; Shi, 1993; Xu et al, 1999; Yang et al, 1990)。近 50 年来, 由于受到人类活动(水域污染、大型水利工程、围垦 等)的影响, 长江口生态环境破坏严重, 影响鱼类种 群的正常繁殖和生长, 例如刀鲚、风鲚、前领间银 鱼等资源量均存在不同程度的下降(Chen et al, 1999; Shi \& Wang, 2002; Zhuang et al, 2006)。以往研究针 对长江口及邻近海区的鱼卵、仔稚鱼群落研究较多 (Jiang et al, 2006; Jiang \& Shen, 2006; Luo et al, 1994; Yang et al, 1990; Zhu et al, 2002), 而涉及河口口内 区域的报道很少(Xu et al, 1999)。作者利用 2005 和 2006 年春、秋季在长江口水域进行的鱼卵、仔稚鱼 调查资料, 并结合 20 世纪 90 年代初, 在该水域调 查的历史资料, 对其种类组成、数量特征的季节和
年际变化进行分析研究, 为河口和近海渔业的可持 续发展及海洋生物资源的科学保护和合理利用提 供参考依据和基础资料。

\section{1 材料与方法}

租用沪南渔 4110 号船只于 2005 年 4 月中旬和 11 月上旬、2006 年 4 上旬和 9 月下旬按照《海洋 调查规范》(GB12763.1-7-91) 对长江河口水域 $\left(\mathrm{E} 121^{\circ} 00^{\prime} \sim 122^{\circ} 10^{\prime} ; \mathrm{N} 31^{\circ} 00^{\prime} \sim 31^{\circ} 50^{\prime}\right.$ ) 进行了 4 个 航次的表层拖网取样调查, 每个航次的站位一致, 每站点在潮水涨喤时取样 3 次, 取平均值作为该站 位的丰度(个/网)(图 1)。拖网时间为 $10 \mathrm{~min}$, 船速一 般保持在 $2.5 \sim 3$ 节。其中, 2005 年的航次同时也进 行了垂直拖网的取样, 在平潮时由底层至表层缓慢 拖曳 $1 \mathrm{~min}$ 左右, 取样 3 次, 取平均值作为该站位的 丰度(个/网)；2005 年 11 月上旬航次因故未调查 S8 和 S9 站点。同时，结合 1990 年 9 月上旬和 1991 年 3 月下旬进行的上海市海岛资源调查中相同站位 (采样方法也相同)的历史数据进行对比分析。

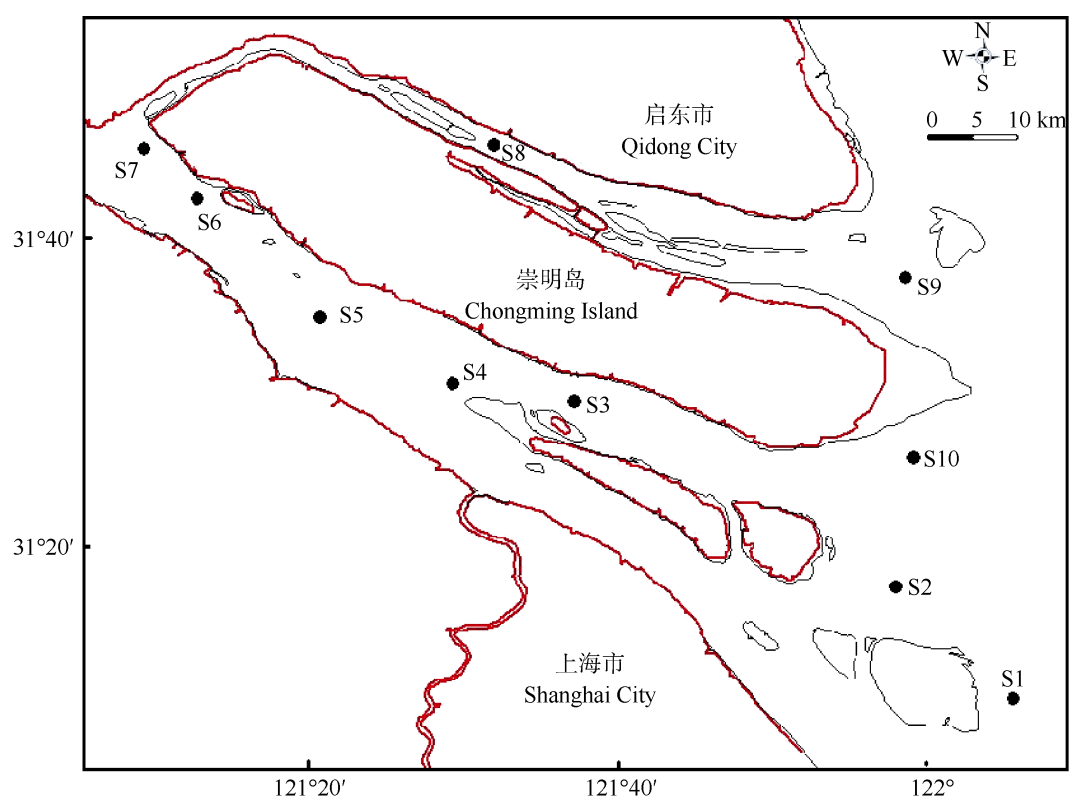

图 1 长江河口水域鱼卵、仔稚鱼调查站位示意图(S1 S10 为调查站位点)

Fig. 1 Survey stations for eggs and larvae in the Yangtze estuary (S1 S10 stations)

采样网具为大型浮游生物网, 网长 $2.8 \mathrm{~m}$, 网口 直径 $0.8 \mathrm{~m}$, 网口面积 $0.5 \mathrm{~m}^{2}$, 篮绢规格 JP12(篎孔 宽 $0.507 \mathrm{~mm}$ )。各个站位的水深为 $3 \sim 8 \mathrm{~m}$ 。 $\mathrm{S} 3 \sim \mathrm{S} 7$ 站点位于河口淡水区，实测盐度低于 $2 \%$; 其他站 点为河口咸淡水区，盐度为 $5 \%$ 18\%。
所采集的样品立即用 $5 \%$ 福尔马林溶液固定, 带回实验室进行种类鉴定、计数和发育阶段的判 别。根据鱼类生活方式划分为 5 种生态类群(Elliott \& Dewailly, 1995)。 


\section{2 结 果}

\section{1 种类组成、生态类群和优势种}

六个航次调查共采集到鱼卵、仔稚鱼 36 种(类), 其中鉴定到种共计 27 种, 5 种标本鉴定到属, 3 种标 本鉴定到科, 1 种标本未定种(表 1)。春季平均种类 数低于秋季, 分别为 6.0 和 9.3(种/网次)。其中, 1990
年 9 月和 1991 年 3 月采集的种(类)数分别为 12 和 1 种; 2005 年 4 月和 11 月为 12 和 3 种; 2006 年 4 月 和 9 月为 6 和 12 种(类)。

采集到仔鱼的种类有 30 种，鱼卵种类有 9 种, 而采集到稚鱼的种类数很低。生态类群以海洋鱼类 种类最多，有 13 种，占总种类数的 $36.1 \%$ ，主要分

表 $11990 、 1991 、 2005$ 和 2006 年长江口水域鱼卵、仔稚鱼的种类、数量百分比 $(\%)$ 和生态类型

Tab. 1 Species, percent and ecological guilds of eggs and larvae in 1990, 1991, 2005 and 2006 in the Yangtze estuary

\begin{tabular}{|c|c|c|c|c|c|c|c|c|}
\hline \multirow{2}{*}{ 种类 Species } & \multicolumn{6}{|c|}{ 出现时间 Time } & \multirow{2}{*}{$\begin{array}{c}\text { 发育阶段 } \\
\text { Developmental stages }\end{array}$} & \multirow{2}{*}{$\begin{array}{c}\text { 生态类型 } \\
\text { Ecological guilds }\end{array}$} \\
\hline & 1990-09 & 1991-03 & 2005-04 & $2005-11$ & 2006-04 & 2006-09 & & \\
\hline 寿南小沙丁鱼 Sardinella zunasi & 0.1 & & & & & & $\mathrm{~L}$ & $\mathrm{C}$ \\
\hline 小沙丁鱼属 sp. Sardinella sp. & & & & & & 3.9 & $\mathrm{~L}$ & $\mathrm{C}$ \\
\hline 鳓鱼 Ilisha elongata & & & 1.9 & & & & EG & $\mathrm{C}$ \\
\hline 鳀 Engraulis japonicus & & & & & & 0.1 & $\mathrm{~L}$ & $\mathrm{C}$ \\
\hline 中领棱鳀 Thrissa mystax & & & 0.9 & & & & EG & $\mathrm{C}$ \\
\hline 凤鲚 Coilia mystus & 37.1 & & & & & 0.8 & $\mathrm{~L} / \mathrm{J}$ & $\mathrm{C}$ \\
\hline 中华小公鱼 Stolephorus chinensis & & & & & & 4.2 & $\mathrm{~L}$ & $\mathrm{C}$ \\
\hline 小公鱼属 sp. Stolephorus sp. & 22.5 & & & & & 2.5 & $\mathrm{EG} / \mathrm{L}$ & $\mathrm{C}$ \\
\hline 太湖新银鱼 Neosalanx taihuensis & & & 1.9 & 94.6 & 24.7 & 19.0 & $\mathrm{~L}$ & B \\
\hline 有明银鱼 Salanx ariakensis & & & & 4.7 & & & $\mathrm{~L}$ & $\mathrm{C}$ \\
\hline 前领间银鱼 Hemisalanx prognathus & & & 2.8 & & 67.9 & & $\mathrm{~L}$ & $\mathrm{D}$ \\
\hline 日本鳗鳎 Anguilla japonica & & & & & 0.1 & & $\mathrm{~L} / \mathrm{J}$ & E \\
\hline 寡鳞飘鱼 Pseudolaubuca engraulis & & & & & & 0.5 & $\mathrm{~L} / \mathrm{J}$ & A \\
\hline 麦穗鱼 Pseudorasbora parva & & & 69.8 & & 0.1 & & $\mathrm{~L}$ & A \\
\hline 翅嘴红鲌 Erythroculter ilishaeformis & 10.3 & & & & & & $\mathrm{~L}$ & A \\
\hline 鲤科 sp. Cyprinidae sp. & & & & & & 0.1 & $\mathrm{~L}$ & A \\
\hline 黄颡鱼 Pelteobagrus fulvidraco & 0.1 & & & & & & $\mathrm{~L}$ & A \\
\hline 食蚊鱼 Gambusia affinis & & & 0.9 & & & & $\mathrm{~L}$ & A \\
\hline 鲻 Mugil cephalus & & & 0.9 & & & & EG & B \\
\hline 白姑鱼 Argyrosomus argentatus & & & & & & 1.7 & $\mathrm{~L}$ & $\mathrm{C}$ \\
\hline 叫姑鱼 Johnius belengeri & & & & & 3.6 & & $\mathrm{~L}$ & $\mathrm{C}$ \\
\hline 棘头梅童鱼 Collichthys lucidus & 2.6 & & & & & & $\mathrm{~L}$ & B \\
\hline 鲷科 sp. Sparidae sp. & & & & & & 66.6 & $\mathrm{EG} / \mathrm{L}$ & $\mathrm{C}$ \\
\hline 细鳞䱨 Therapon jarbua & & 100 & & & & & $\mathrm{~L}$ & $\mathrm{C}$ \\
\hline 短魾齿鱼 Champsodon snyderi & & & 5.7 & & & & EG & $\mathrm{C}$ \\
\hline 纹缟虾虎鱼 Tridentiger trigonocephalus & & & & & & 0.8 & $\mathrm{~L}$ & B \\
\hline 虾虎鱼科 sp. Gobiidae sp. & 17.7 & & 2.5 & 0.7 & 3.6 & & $\mathrm{~L}$ & B \\
\hline 栉孔虾虎鱼属 sp. Ctenotrypanchen sp. & 0.1 & & & & & & $\mathrm{~L}$ & B \\
\hline 乌鳢 Channa arga & 0.1 & & & & & & $\mathrm{~L}$ & A \\
\hline 蓝点马鲛 Scomberomorus koreanus & & & 1.9 & & & & EG & $\mathrm{C}$ \\
\hline 圆尾斗鱼 Macropodus chinensis & 0.1 & & & & & & $\mathrm{~L}$ & A \\
\hline 条鳎属 sp. Zebrias sp. & & & 0.9 & & & & EG & $\mathrm{C}$ \\
\hline 宽体舌鳎 Cynoglossus robustus & & & & & & 0.1 & $\mathrm{~L}$ & $\mathrm{C}$ \\
\hline 窄体舌鳎 Cynoglossus semilaevis & 0.7 & & & & & & $\mathrm{EG} / \mathrm{L}$ & B \\
\hline 舌鲳属 sp. Cynoglossus sp. & & & 15.1 & & & & $\mathrm{~L}$ & \\
\hline 未定种 1 种 unidentified & 8.4 & & & & & & $\mathrm{~L}$ & \\
\hline
\end{tabular}

生态类群: $\mathrm{A}$, 淡水鱼类; B, 咸淡水鱼类; C, 海洋鱼类; D, 溯河洄游鱼类; E, 降海洄游鱼类. 发育阶段: EG, 鱼卵; L, 仔鱼; J, 稚鱼。

Ecological guilds: A, Fresh water fish; B, Brackish fish; C, Marine fish; D, Anadromous fish; E, Catadromous fish. Developmental stages: EG, Eggs; L, Larvae; J, Juvenile. 
布在口门区 $(\mathrm{S} 1 、 \mathrm{~S} 2 、 \mathrm{~S} 9$ 和 S10 站点); 其次为淡水 鱼类, 共 8 种, 占总种数的 $22.2 \%$, 主要有翅嘴红鲌 (Erythroculter ilishaeformis)、麦穗鱼(Pseudorasbora parva)、寡鳞飘鱼(Pseudolaubuca engraulis)等, 主要分 布在长江口南支水域( $\mathrm{S} 3 \sim \mathrm{S} 7$ 等站点); 咸淡水鱼类 和过河口性鱼类分别为 7 和 3 种，占 $19.4 \%$ 和 $7.1 \%$, 主 要有太湖新银鱼(Neosalanx taihuensis)、凤鲚(Coilia mystus)、前领间银鱼(Hemisalanx prognathus)等。

以占各站总数量的比例 $>20 \%$ 的种类定义为优 势种, 1990 年 9 月凤魰为绝对优势种, 数量百分比 为 $37.1 \%$; 其次为小公鱼属 sp., 数量百分比为 $22.5 \%$ 。2005 年 4 月麦穗鱼为优势种, 数量百分比 为 $69.8 \% ; 11$ 月太湖新银鱼为优势种, 数量百分比 为 $94.6 \%$, 在各个站位的出现频次也较高。2006 年 4 月前, 领间银鱼为优势种, 数量百分比为 $67.9 \%$; 9
月优势种为鲷科鱼类和太湖新银鱼(表 1)。

\section{2 数量分布}

6 次调查共发现鱼卵 727 粒，仔稚鱼 852 尾，其 中垂直网采集到鱼卵 12 粒和仔稚鱼 78 尾。19901991 年春秋季在表层拖网中共发现鱼卵 2 粒, 仔稚 鱼 215 尾; 2005 年春秋季共发现鱼卵 11 粒，仔稚鱼 271 尾; 2006 年发现鱼卵 702 粒，仔稚鱼 288 尾。春 季调查的 3 个航次所捕获的鱼卵、仔稚鱼总个体数 均低于秋季 3 个航次，分别是 4、43、11 和 731、 296、979 个。在前 4 个航次里，垂直网和表层拖网 采集到的鱼卵总数相差不大, 而仔稚鱼数量前者仅 为后者的 $8 \%$, 且前者采集到的种类数均低于后者 (表 2)。一般来说, 不论表层拖网还是垂直拖网, 均 是鱼卵数低于仔稚鱼数, 但 2006 年 9 月采集的鱼卵 数高于仔稚鱼数, 约是后者的 2.5 倍。

表 2 不同调查航次所捕获的鱼卵和仔稚鱼的总个体数和种类数

Tab. 2 Total number of individuals and species of egg, larvae and juvenile in different surveys

\begin{tabular}{|c|c|c|c|c|c|c|}
\hline \multirow{2}{*}{$\begin{array}{l}\text { 时间 } \\
\text { Time }\end{array}$} & \multicolumn{2}{|c|}{ 垂直拖网 Vertical trawl } & \multirow{2}{*}{$\begin{array}{l}\text { 种类数 } \\
\text { Number of } \\
\text { Species }\end{array}$} & \multicolumn{2}{|c|}{ 表层拖网 Horizontal trawl } & \multirow{2}{*}{$\begin{array}{c}\text { 种类数 } \\
\text { Number of } \\
\text { Species }\end{array}$} \\
\hline & 鱼卵 Egg & 仔稚鱼 Larvae and juvenile & & 鱼卵 Egg & 仔稚鱼 Larvae and juvenile & \\
\hline 1990.09 & 10 & 24 & 7 & 2 & 212 & 12 \\
\hline 1991.03 & 0 & 0 & 0 & 0 & 3 & 1 \\
\hline 2005.04 & 2 & 0 & 2 & 11 & 29 & 12 \\
\hline 2005.11 & 0 & 54 & 2 & 0 & 242 & 3 \\
\hline 2006.04 & - & - & - & 0 & 11 & 6 \\
\hline 2006.09 & - & - & - & 702 & 277 & 12 \\
\hline
\end{tabular}

根据表层拖网的丰度数据进行鱼卵和仔稚鱼 的平均丰度分布差异分析。各个航次的鱼卵出现的 站位数均显著低于仔稚鱼(ANOVA, $P<0.01$ )(图 2)。 鱼卵丰度最高值的出现在 S9 站点, 2006 年 9 月高达 700 个/网(图 2f), 绝大多数为鲷科鱼类鱼卵, 少数 为小公鱼属鱼卵; 仔稚鱼丰度最高值出现在 S3 站 点, 2005 年 11 月达 179 个/网(图 2d), 主要为太湖新 银鱼仔鱼。总体来讲, 6 个航次中 $\mathrm{S} 9$ 站点采集的鱼 卵和仔稚鱼平均丰度最高, 为 138.8 个/网/航次; 其 次为 $\mathrm{S} 3$ 站点, 为 40.5 个/网/航次, 其它站点一般为 $4.3 \sim 15.2$ 个/网/航次。6 个航次中, 河口咸淡水的 5 个站点 $(\mathrm{S} 1 、 \mathrm{~S} 2 、 \mathrm{~S} 8 、 \mathrm{~S} 9$ 和 $\mathrm{S} 10)$ 的鱼卵和仔稚鱼总 数分别为 715 粒和 357 尾, 而南支淡水区 5 个站点 $(\mathrm{S} 3 \sim \mathrm{S} 7)$ 为 0 粒和 495 尾。与 1990-1991 年数据相 比, 2005-2006 年春、秋季的鱼卵和仔稚鱼的平均 丰度均有所增加(图 2)。

\section{3 讨 论}

本研究的六次调查虽未在 5-8 月鱼卵、仔稚 鱼补充高峰期进行, 但调查到的种类达到了 36 种 (类)，略高于世界上同纬度地区其它河口或泻湖， 如南非的 East Kleinemond 泻湖 $\left(33^{\circ} \mathrm{S}, 33\right.$ 种)(Cowley \& Whitfield, 2001), 澳大利亚的 Nornalup-Walpole 江湖(S35 ${ }^{\circ}, 31$ 种)(Potter \& Hyndes, 1994)和 Río de la Plata 河口(S35, 35 种)(Lasta, 1995; Macchi et al, 2002), 说明长江河口鱼卵、仔稚鱼的物种多样性较高, 是 河口及邻近海洋渔业资源的重要补充来源之一。

Yang et al(1990)对长江口及邻近海域鱼卵、仔 稚鱼进行了周年调查发现, 春季 3-4 月上旬鱼卵 和仔稚鱼数量较低, 略低于秋季, 这与本研究结果 类似。本研究发现春季航次鱼卵、仔稚鱼的总个体 数均低于秋季航次(表 2), 说明春季(3-4 月初)产卵 

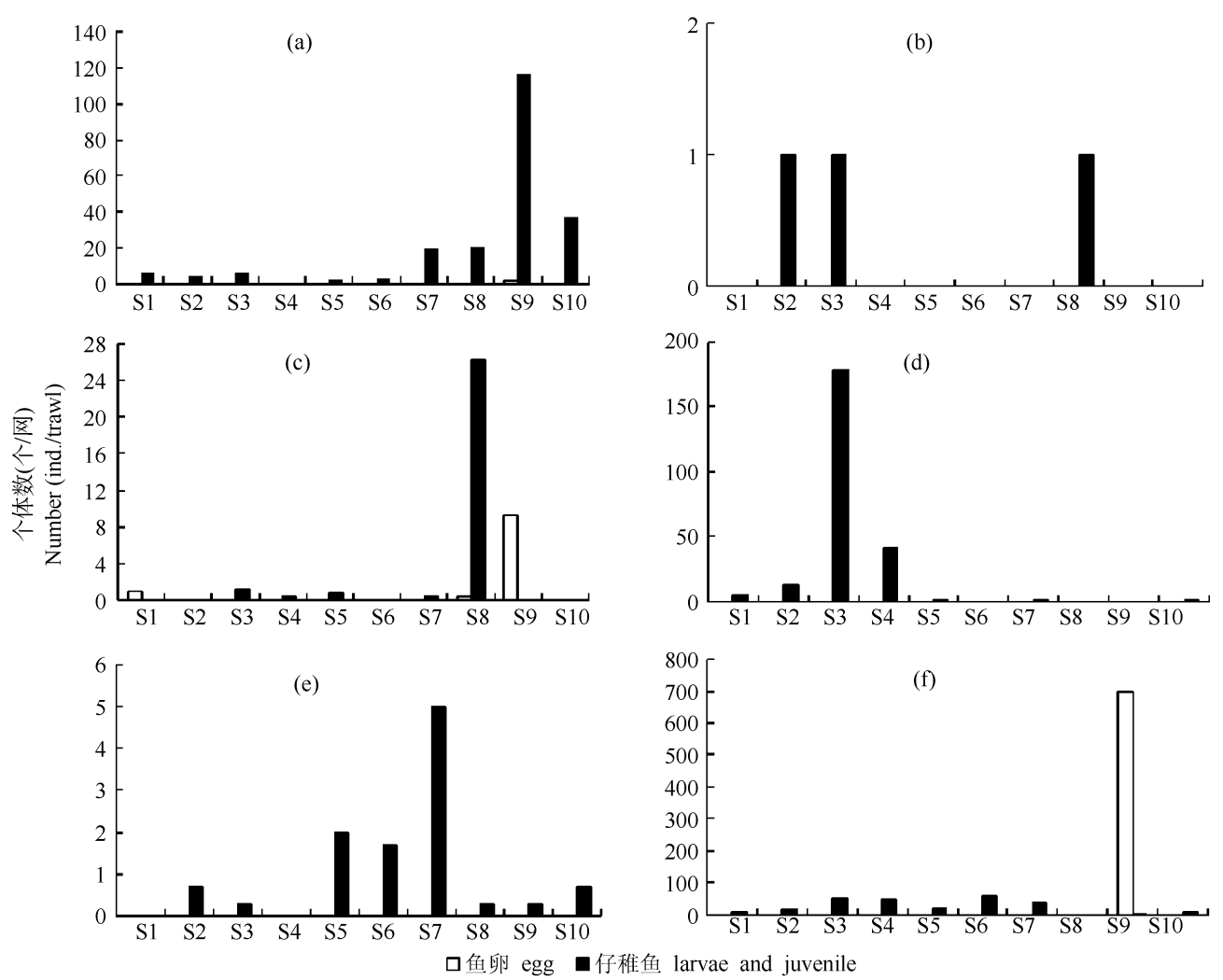

图 2 不同调查航次鱼卵、仔稚鱼(表层拖网)的平均丰度分布(个/网)

Fig. 2 Distribution of average abundance of eggs, larvae and juveniles for different surveys (ind./trawl) (a): 1990-09; (b)1991.03; (c): 2005-04; (d): 2005-11; (e): 2006-04; (f): 2006-09.

的鱼类种类较少。大量研究表明，口内淡水区鱼卵 数量分布很少, 而口门咸淡水区及邻近海域分布较 多(Xu et al, 1999; Yang et al, 1990; Zhu et al, 2002)。 本研究的 6 次调查均未在河口淡水区 (S3 S7 站点) 采集到鱼卵，而在河口咸淡水区(S1 和 S9站点)则采 集到较多鱼卵，主要因淡水鱼类多产黏性和沉性卵， 而口门区许多海洋鱼类产漂浮性卵(Zhuang et al, 2006), 有利于大型浮游生物网捕获。

长江口鱼卵、仔稚鱼群落的另一特点是淡水鱼 类种类较多, 约占总种数的三分之一, 而邻近海域 则以海洋鱼类种类最多, 淡水鱼类比例很少(Jiang et al, 2006; Jiang \& Shen, 2006; Yang et al, 1990; Zhu et al, 2002), 这也反映了长江口鱼卵、仔稚鱼群落组 成具有一定的特殊性。

许多研究表明, 近 20 年来, 由于受到过度捕 捞、水域环境污染等人为因素影响, 长江河口鱼类 资源衰退严重，各种鱼类的补充群体资源量下降迅 速, 这必将导致鱼卵、仔稚鱼种类组成发生较大变 化(Xu et al, 1999; Zhuang et al, 2006)。本研究发现
1990 和 2006 年 9 月航次的种类组成差异较大, 如 1990 年尧嘴红鲌数量和出现频次均较高, 而 2006 年没有发现尧嘴红鲌，优势种为太湖新银鱼，而小 公鱼属的丰度差异相对较小(表 1)。另外, 2006 年调 查的风鲚的仔稚鱼平均丰度仅约是 1990 年的 $1 / 10$, 说明近十几年来风鲚的补充群体资源已明显下降, 在一定程度上解释了风鲚成鱼资源有所下降的原 因。前领间银鱼的补充群体更是严重下降, Xu et al(1999)于 1997 年 5 月在长兴岛南侧未发现前领间 银鱼的仔稚鱼, 本研究调查到的丰度也很低, 仅为 1.3 个/网，低于 1986 年同期的 14.5 个/网(Huang et al, 1992), 说明该种群的补充群体已降到很低的水平, 造成其成鱼资源在近 20 年来一直处于很低的水平, 主要原因有亲体的过度捕捞和产卵场水域的严重 污染等(Xu et al, 1999; Ni et al, 1990)。

综上所述, 长江河口鱼卵、仔鱼群落组成已发 生了较大变化, 主要是部分经济鱼类的补充群体下 降较为严重, 但由于相关研究报道得很少, 进一步 深入研究和相关保护工作急需加强。 
致谢：本次调查得到崇西湿地科学实验站和华 东师范大学河口海岸学重点实验室同仁的大力帮 助; 种类鉴定得到了东海水产研究所陈莲芳研究

\section{参考文献:}

Barnes RSK. 1974. Estuarine Biology[M]. London: Edward Arnold. Bell JD, Pollard DA, Burchmore JJ, Pease BC, Middleton MJ. 1984. Structure of a fish community in a temperate tidal mangrove creek in Botany Bay, New South Wales[J]. Aust J Mar Freshw Res, 35(1): 33-46.

Berasategui AD, Acha EM, Fernández Araoz NC. 2004. Spatial patterns of ichthyoplankton assemblages in the Río de la Plata Estuary (Argentina-Uruguay)[J]. Est Coast Shelf Sci, 60(4): 599-610.

Chen YQ, Chen YQ. 1999. The new and sustainable utilization pattern on the fishery resources in the Yangtze estuary[J]. J Fish Sci Chn, 6(5): 72-74. [陈亚翟，陈渊泉. 1999. 长江河口区渔业资源利用新模式及 可持续利用的探讨 [J]. 中国水产科学, 6(5): 72-74.]

Chen YQ, Jiang M, Han JD. 1999. A study on the temporal and spatial distributional features of juvenil Anguilla japonica and utilizing status of the resource in the Changjiang estuary[J]. J Fish Sci Chn, 5(S1): 37-40. [陈渊泉, 蒋玫, 韩金娣. 1999. 长江口鳗苗时空分布特征及 资源利用现状研究 [J]. 中国水产科学, 5(S1): 37-40.]

Cowley PD, Whitfield AK. 2001. Ichthyofaunal characteristics of a typical temporarily open/closed estuary on the Southeast coast of South Africa[J]. Ichthyol Bull. (71):1-17.

Deegan LA. 1990. Effects of estuarine environmental conditions on population dynamics of young-of-the-year gulf menhaden[J]. Mar Ecol Prog Ser, 68(1-2): 195-205.

Elliott M, Dewailly F. 1995. The structure and components of European estuarine fish assemblages[J]. Nether J Aquatic Ecol, 29(3-4): 397-417.

Haedrich RL, Haedrich SO. 1974. A seasonal survey of the fishes in the mystic river, a polluted estuary in downtown Boston, Massachusetts[J]. Estuar Cstl Mar Sci, 2(1): 59-73.

Huang JB. 1988. Study on the relationship on quantity between eggs and larvae or juveniles in the Yangtze River estuary[J]. Fish Sci Technol Inform, (1): 20-21. [黄晋彪. 1988. 长江河口区鱼卵和仔、稚鱼种间 数量关系的研究 [J]. 水产科技情报, (1): 20-21.]

Jiang M, Shen XQ. 2006. Abundance distributions of pelagic fish eggs and larva in the Changjiang River estuary and vicinity waters in summer[J]. Mar Sci, 30(6): 92-97. [蒋玫, 沈新强. 2006. 长江口及邻近水域夏季 鱼卵、仔鱼数量分布特征 [J]. 海洋科学, 30(6): 92-97.]

Jiang M, Shen XQ, Chen LF. 2006. Relationship between with abundance distribution of fish eggs, larvae and environmental factors in the Changjiang Estuary and vicinity waters in spring[J].Mar Envuron Sci, 25(2): 37-44. [蒋玫, 沈新强, 陈莲芳. 2006. 长江口及邻近水域春季 鱼卵仔鱼分布与环境因子的关系 [J]. 海洋环境科学, 25(2): 37-44.]

Lasta CA. 1995. La Bahía Samborombón: Zona De Desove Y Cría De Peces[D]. Ph.D. dissertation, Facultad de Ciencias Naturales y Museo, Universidad Nacional de la Plata, La Plata.
员、黄海水产研究所万瑞景研究员和上海海洋大学 钟俊生教授的鼎力相助，谨致谢忱!

Loneragan NR, Potter IC, Lenanton RCJ. 1989. Influence of site, season and year on contributions made by marine, estuarine, diadromous and freshwater species to the fish fauna of a temperate Australian estuary[J]. Mar Biol, 103(4): 461-479.

Macchi GJ, Acha EM, Lasta CA. 2002. Reproduction of black drum (Pogonias cromis) in the Río de la Plata estuary, Argentina[J]. Fish Res, 59(1-2): 83-92.

McErlean AJ, O'Connor SG, Mihursky JA, Gibson CI. 1973. Abundance, diversity and seasonal patterns of estuarine fish populations[J]. Estuar Cstl Mar Sci, 1(1): 19-36.

Ni Y, Wang YH, Xu CY, Xiong GQ. 1990. The Fishes of Shanghai Area[M]. Shanghai: Science and Technology Press in Shanghai. [倪勇, 王幼槐, 许成玉, 熊国强. 1990. 上海鱼类志 $[\mathrm{M}]$. 上海: 上海科学技术出版 社.]

Potter IC, Hyndes GA. 1994. Composition of the fish fauna of a permanently open estuary on the southern coast of Australia, and comparisons with a nearby seasonally closed estuary[J]. Mar Biol, 121(2): 199-209.

Shi DL. 1993. Utilization and management on Japanese eel of Chongming Island of Yangtze estuary[J]. J Mar Fish Chn, (6): 264-266. [施德龙. 1993. 崇明对长江鳗苗资源的利用及管理 [J]. 海洋渔业, (6): 264-266.]

Shi WG, Wang B. 2002. Status quo of tapertail anchovy resource in the estuaries of the Yangtze River[J]. Acta Hydrobiol Sin, 26(6): 648-653. [施炜纲, 王博. 2002. 长江河口区风鲚的资源现状 [J]. 水生生物学 报, 26(6): 648-653.]

Xu ZL, Yuan Q, Jiang M, Zang ZJ. 1999. An investigation of fish eggs, larvae and juveniles in the Changjiang esturary[J]. J Fish Sci Chn, 6(5): 63-64. [徐兆礼, 袁骐, 蒋玫, 藏增加. 1999. 长江口鱼卵和仔、稚鱼 的初步调查[J]. 中国水产科学, 6(5): 63-64.]

Yang DL, Wu GZ, Sun JR. 1990. The investigation of pelagic eggs, larvae and juveniles of fishes at the mouth of the Changjiang River and adjacent areas[J]. Oceanol Limnol, 21(4): 346-355. [杨东莱, 吴光宗, 孙继仁. 1990. 长江口及其邻近海区的浮性鱼卵和仔稚鱼的生态研 究 $[\mathrm{J}]$. 海洋与湖沼, 21(4): 346-355.]

Zhu XH, Liu D, Sha XK. 2002. Relationship between community structure of ichthyoplankton and environmental factors in the estuary of Changjiang river in spring[J]. Bull Mar Sci, 44: 169-178. [朱金华, 刘 栋, 沙学绅. 2002. 长江口春季鱼类浮游生物群落结构与环境因子 的关系 [J]. 海洋科学集刊, 44: 169-178.]

Zhuang P, Wang YH, Li SF, Deng SM. 2006. Fishes of the Yangtze Estuary[M]. Shanghai: Science and Technology Press in Shanghai, 1-100. [庄平, 王幼槐, 李圣法, 邓思明. 2006. 长江口鱼类[M]. 上 海: 上海科学技术出版社, 1-100. 